\title{
RELATO DE CASO DE SECREÇÃO ECTÓPICA DE HORMÔNIO ADRENOCORTICOTRÓFICO: UM DESAFIO DIAGNÓSTICO
}

\author{
A CASE REPORT OF ECTOPIC ADRENOCORTICOTROPIC HORMONE SECRETION: A DIAGNOSTIC CHALLENGE
}

Isabella Santiago de Melo Miranda e Monalisa Ferreira Azevedo.

DOI - 10.5935/2236-5117.2015v52n3/4a08

\section{RESUMO}

A secreção ectópica de hormônio adrenocorticotrófico representa cerca de $5 \%$ a $10 \%$ dos casos de síndrome de Cushing ACTH-dependente. Os tumores carcinoides brônquicos são os mais prevalentes. Os autores relatam o caso de um doente do sexo masculino com hipercortisolismo grave associado a hipocalemia. Depois de extensa investigação, foi diagnosticado tumor carcinoide brônquico, e o paciente foi submetido a tratamento cirúrgico. Cursou no período pósoperatório com insuficiência suprarrenal. Atualmente, encontra-se sem sinais clínicos ou bioquímicos de recidiva da doença. O caso relatado mostra a importância do reconhecimento da secreção ectópica da suprarrenal em um doente com hipercortisolismo endógeno grave associado a hipocalemia, bem como expõe as dificuldades no diagnóstico e no manejo desses doentes.

Palavras-chave. Síndrome de Cushing; tumores carcinoides; hipercortisolismo; diagnostico; tratamento

\section{ABSTRACT}

Ectopic adrenocorticotropic hormone secretion accounts for $5 \%$ to $10 \%$ of ACTH-dependent Cushing's syndrome. Bronchial carcinoid tumors are the most prevalent tumors. The authors report a case of a male patient with severe hypercortisolism associated with hypokalemia. During the investigation, a bronchial carcinoid tumor was diagnosed and the patient was submitted to surgery and evolved in the postoperative period with adrenal insufficiency. Currently, there are no clinical or biochemical signs of recurrence of the disease. The reported case shows the importance in the recognition of the ectopic secretion of adrenocorticotropic hormone in a patient exhibiting severe endogenous hypercortisolism associated with hypokalemia, as well as the difficulties in the diagnosis and management of these patients.

Key words. Cushing's syndrome; carcinoid tumors; hypercortisolism; diagnosis; treatment.
Isabella Santiago de Melo Miranda - médica, endocrinologista, Serviço de Endocrinologia, Hospital Universitário de Brasília (HUB), Universidade de Brasília (UnB)

Monalisa Ferreira Azevedo - médica, endocrinologista, doutora, pós-doutorado na National Institutes of Health - United States of America, Serviço de Endocrinologia, Hospital Universitário de Brasília (HUB/UnB)

Correspondência: Monalisa Ferreira Azevedo. Serviço de Endocrinologia, Hospital Universitário de Brasília (HUB/UnB), Setor de Grandes Áreas Norte 605, CEP 70840-901, Brasília-DF.

Internet: monalisaazevedo@hotmail.com

\section{INTRODUÇÃO}

A síndrome de Cushing compreende um conjunto de sinais e sintomas secundários ao excesso de cortisol circulante, que pode ocorrer devido ao uso crônico de glicocorticoides ou, menos frequentemente, por hiperprodução endógena. ${ }^{1,2} \mathrm{O}$ hipercortisolismo crônico está associado a diversas comorbidades que podem aumentar o risco de elevação da taxa de mortalidade, principalmente cardiovascular. ${ }^{1}$

A síndrome de Cushing endógena é classificada como ACTH-dependente - quando existe hipersecreção desse hormônio com origem em um tumor hipofisário ou a partir de uma fonte ectópica, que leva a um estímulo excessivo das suprarrenais com consequente hipersecreção de cortisol -, ou ACTH-independente, quando o hipercortisolismo se deve a hipersecreção autônoma de uma ou de ambas as suprarrenais. A secreção ectópica de hormônio adrenocorticotrópico (ACTH) ou corticotropina representa cerca de $5 \%$ a $10 \%$ dos casos de síndrome de Cushing ACTH-dependente. ${ }^{2}$

A maioria desses casos ocorre devido a neoplasias intratorácicas, e os tumores carcinoides são mais prevalentes. $^{3}$ 
A investigação diagnóstica e o tratamento representam um desafio na prática clínica, uma vez que, em até $50 \%$ dos casos, a fonte de secreção de hormônio adrenocorticotrópico pode não ser identificada por meio de exames de imagem e ainda pode permanecer oculta. ${ }^{2}$

\section{RELATO DO CASO}

Paciente do sexo masculino, com 43 anos de idade, foi admitido no pronto-socorro com baixo grau de consciência, pico hipertensivo e hipocalemia grave $(K=2$ $\mathrm{mEq} / \mathrm{L})$. Depois de receber tratamento de suporte, houve correção do teor do potássio, normalização da pressão arterial e restabelecimento do estado de consciência. Previamente hígido, o doente referiu ter iniciado quadro de fraqueza muscular proximal progressiva, escurecimento da pele, edema de face e ganho ponderal progressivo três meses antes da internação. Negou uso prévio de medicamentos. Ao exame físico apresentou fácies em lua-cheia, hiperpigmentação cutânea e de mucosa oral, distribuição centrípeta de gordura e estrias violáceas no abdome.

Os exames laboratoriais revelaram elevada concentração de cortisol livre urinário em dois momentos distintos (4838 mcg/24 h e 1939,5 mcg/24h - valor de referência 20,9 a 292,3 mcg/24 h). Não houve supressão do cortisol após uso de $1 \mathrm{mg}$ de dexametasona $(46,61 \mathrm{mcg} / \mathrm{dL}$ - valor de referência até $1,8 \mathrm{mcg} / \mathrm{dL}$ ). Posteriormente, foram coletadasduasamostras dehormônioadrenocorticotrópico que vieram elevadas $(202 \mathrm{pg} / \mathrm{mL}$ e $410 \mathrm{pg} / \mathrm{mL}$ - valor de referência até $46 \mathrm{pg} / \mathrm{mL}$ ). 0 teste de supressão com alta dose de dexametasona mostrou queda de $18 \%$ em relação ao cortisol basal, sugerindo tratar-se de doença ectópica, com cortisol basal de $51,25 \mathrm{mcg} / \mathrm{dL}$ e cortisol de $41,8 \mathrm{mcg} /$ dL após $8 \mathrm{mg}$ de dexametasona. 0 cateterismo bilateral de seios petrosos inferiores mostrou gradientes, central e periférico, de hormônio adrenocorticotrópico basal de 1,02 e após administração de desmopressina de 1,18, ambos sugestivos de doença periférica (tabela). A tomografia de tórax com contraste evidenciou nódulo pulmonar no segmento basal posterior à direita, não calcificado e de contornos espiculados, com 7,5 mm de extensão (figura).

Iniciou-se a administração de cetoconazol para controle do hipercortisolismo até a intervenção cirúrgica. Porém, após duas semanas de uso, o doente teve elevação do teor das transaminases (ALT de $318 \mathrm{U} / \mathrm{L}$ - valor de referência 10 a $49 \mathrm{U} / \mathrm{L}$, e AST de $57 \mathrm{U} / \mathrm{L}$ - valor de referência até $34 \mathrm{U} / \mathrm{L}$ ) e de gama-GT (917 U/L - valor de referência até $73 \mathrm{U} / \mathrm{L}$ ), e a medicação foi suspensa. As alterações desapareceram algumas semanas depois da suspensão do cetoconazol.

O paciente foi submetido a nodulectomia no lobo inferior do pulmão direito. 0 exame anatomopatológico mostrou tumor carcinoide típico com margens cirúrgicas livres. No período pós-operatório imediato, o paciente teve insuficiência suprarrenal com cortisol de $2 \mathrm{mcg} /$ $\mathrm{dL}$ (valor de referência 5,27 a 22,45 mcg/dL) e teor do hormônio adrenocorticotrópico de $17 \mathrm{pg} / \mathrm{mL}$ (valor de referência até $46 \mathrm{pg} / \mathrm{mL}$ ). Necessitou de reposição com glicocorticoide (prednisona $5 \mathrm{mg} / \mathrm{dia}$ ).

Durante o acompanhamento, dois anos depois da cirurgia, o doente manteve-se sem sinais clínicos ou bioquímicos de recidiva da doença.

Tabela. Cateterismo de seios petrosos inferiores com valores de concentração do hormônio adrenocorticotrópico basal e após administração de desmopressina em veia periférica e seios petrosos inferiores direito e esquerdo

\begin{tabular}{|lccccc|}
\hline Hormônios & \multicolumn{5}{c}{ Tempo (minuto) } \\
& 0 & 3 & 5 & 10 \\
\hline ACTH ${ }^{*}$ periférico & 127 & 140 & 119 & 121 \\
\hline ACTH central (direito) & 130 & 133 & 141 & 133 \\
\hline ACTH central (esquerdo) & 115 & 151 & 139 & 143 \\
\hline
\end{tabular}

${ }^{*} \mathrm{ACTH}$ : hormônio adrenocorticotrópico

Valores de hormônio adrenocorticotrópico: pg/mL

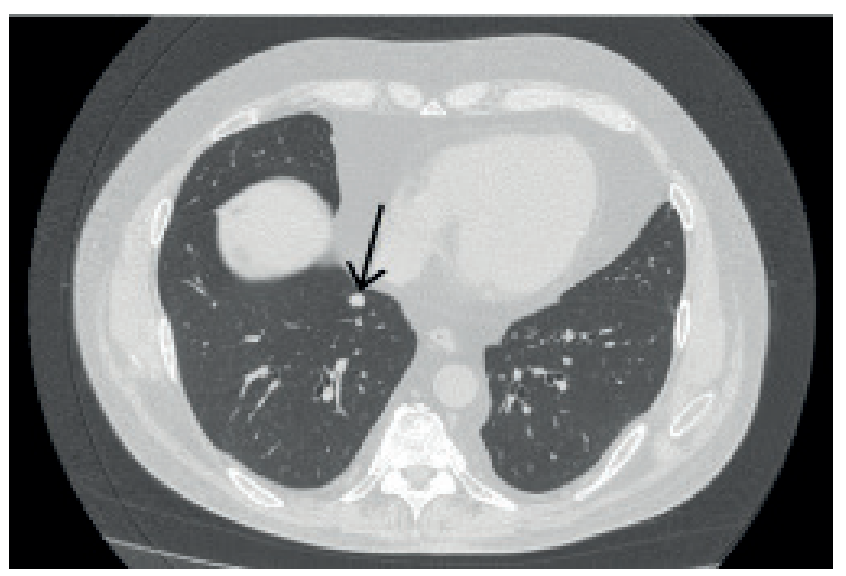

Figura. Tomografia de tórax do paciente em referência, com contraste, corte axial. A seta em branco aponta o nódulo no segmento basal inferior do pulmão direito

\section{DISCUSSÃO}

A síndrome de Cushing endógena é uma doença rara, caracterizada pela exposição crônica a altos teores de glicocorticoides. ${ }^{4} \mathrm{~A}$ principal causa da síndrome de Cushing ACTH-dependente é a doença de Cushing ou adenoma hipofisário secretor de hormônio adrenocorticotrópico, responsável por aproximadamente $85 \%$ a $90 \%$ dos casos. A secreção ectópica do hormônio em estudo corresponde aos $5 \%$ a $10 \%$ restantes. ${ }^{3,5}$ 
O diagnóstico da síndrome de Cushing endógena envolve três etapas, ou seja, confirmação bioquímica do hipercortisolismo, definição etiológica (ACTHdependente ou independente) e, nos casos de ACTHdependente, a diferenciação entre doença central (hipofisária) e periférica. ${ }^{4,6}$

Pacientes com secreção ectópica de hormônio adrenocorticotrópico geralmente apresentam concentrações desse hormônio mais elevadas do que os casos com doença de Cushing (> $200 \mathrm{pg} / \mathrm{mL}$ ), ${ }^{3}$ como foi evidenciado no caso relatado. 0 hormônio em referência é derivado da clivagem da pró-opiomelanocortina, que também resulta na síntese do hormônio alfa-MSH. Quando presente em excesso na circulação, esse peptídeo irá se ligar a seu receptor MCR1 na pele e estimula os melanócitos, o que causa hiperpigmentação cutaneomucosa. $^{7}$

Tumores secretores de hormônio adrenocorticotrópico ectópico costumam não responder ao teste de supressão com dose elevada de dexametasona ou após estímulo com fator ou hormônio liberador de corticotropina (CRH) ou desmopressina, ao contrário do observado nos adenomas hipofisários. Entretanto, alguns carcinoides brônquicos podem apresentar resposta a esses testes dinâmicos, num padrão idêntico ao observado nos adenomas hipofisários, o que dificulta o esclarecimento diagnóstico. Devido a essa baixa acurácia dos testes dinâmicos não invasivos, acredita-se que a combinação entre eles possa aumentar a sensibilidade para o diagnóstico diferencial entre doença de Cushing e secreção ectópica de hormônio adrenocorticotrópico. ${ }^{8}$

0 cateterismo de seios petrosos inferiores, quando realizado em centros especializados, é considerado exame padrão-ouro para o diagnóstico diferencial entre doença hipofisária e secreção ectópica do hormônio em foco. ${ }^{4}$ Mas é importante lembrar que é um exame invasivo com potenciais riscos de complicação e nem sempre é disponível.

A secreção ectópica de hormônio adrenocorticotrópico deve ser suspeitada diante do doente com hipercortisolismo grave e hipocalemia, ${ }^{6,9}$ como no caso relatado. 0 excesso de glicocorticoides leva à saturação da enzima 11-beta-hidroxiesteroide desidrogenase do tipo 2 nos rins, responsável por fazer a conversão do cortisol em cortisona (forma inativa). Em casos de hipercortisolismo grave, há maior concentração de cortisol disponivel para se ligar ao receptor mineralocorticoide nos rins, resultando em quadro de hipocalemia e acidose metabólica. ${ }^{10}$

Diversos tumores podem estar associados a secreção ectópica de hormônio adrenocorticotrópico, como o carcinoma pulmonar de pequenas células, os tumores carcinoides de pulmão, timo e trato gastrointestinal, o feocromocitoma e o carcinoma medular de tireoide. Os tumores carcinoides brônquicos parece serem os mais frequentes. ${ }^{6}$ A fonte de secreção ectópica do hormônio adrenocorticotrópico pode permanecer, porém, sem ser identificada durante anos em grande parte dos casos. ${ }^{11}$

Os carcinoides brônquicos geralmente são pequenos e, portanto, podem não ser identificados em exames convencionais de imagem. Entretanto, em quase todos os casos podem ser localizados por meio de exame de tórax com uso de tomografia computadorizada ou de ressonância magnética. ${ }^{6}$ Alguns doentes podem precisar de fazer uso de inibidores da esteroidogênese (metirapona ou cetoconazol) para controle do hipercortisolismo até a realização da conduta cirúrgica, ${ }^{10}$ como foi o caso do doente em estudo, que fez uso de cetoconazol. Sabe-se do risco de hepatotoxicidade com o uso desse fármaco, ${ }^{2}$ como ocorreu com esse paciente, o qual apresentou elevação das transaminases e do valor do gama-GT, necessitando-se interromper o uso do medicamento. Depois da suspensão do cetoconazol, os resultados dos exames retornaram à faixa de normalidade.

Uma vez localizada a lesão suspeita, o tratamento de escolha consiste na remoção do tumor. ${ }^{12} A$ remissão da doença pode ocorrer em até $80 \%$ dos pacientes operados. ${ }^{6}$

Os tumores carcinoides brônquicos podem estar associados a secreção ectópica de hormônio adrenocorticotrópico em $1 \%$ a $5 \%$ dos casos. ${ }^{13}$ Ainda existem poucos estudos sobre o comportamento e o prognóstico desses tumores. Um estudo publicado em 2012 mostrou retrospectivamente a avaliação de quatorze pacientes submetidos a ressecção de tumores carcinoides brônquicos associados à síndrome de Cushing. Esses indivíduos foram acompanhados por, em média, 59 meses após o procedimento cirúrgico e nenhum apresentou recorrência do tumor ou recidiva do hipercortisolismo durante o período de acompanhamento. ${ }^{13} \mathrm{O}$ doente aqui reportado está, há dois anos, sem sinais clínicos ou bioquímicos de recidiva da doença.

Em suma, o caso relatado aborda a importância do reconhecimento da secreção ectópica de hormônio adrenocorticotrópico perante um doente com hipercortisolismo endógeno grave associado a hipocalemia. Além disso, são expostos os desafios envolvidos no diagnóstico, que contemplam não só a confirmação bioquímica do hipercortisolismo como também determinar a causa e a localização da lesão secretora. 


\section{REFERÊNCIAS}

1. Lacroix A, Feelders RA, Stratakis CA, Nieman LK. Cushing's syndrome. Lancet. 2015;386(8):913-27. DOI: 10.1016/ S0140-6736(14)61375-1.

2. Vieira-Corrêa M, Moroto D, Carpentieri G, Veras I, Kater CE. The 4Ds of ectopic ACTH syndrome: diagnostic dilemmas of a difficult disease. Arq Bras Endocrinol Metab. 2019;63(2):17581. DOI: $10.20945 / 2359-3997000000129$.

3. Salgado LR, Candida, M, Fragoso BV, Knoepfelmacher $M_{\text {, }}$ Machado MC, Domenice $S$ et al. Ectopic ACTH syndrome: our experience with 25 cases. Eur J Endocrinol. 2006;155(5):72533. DOI: 10.1530/eje.1.02278.

4. Nieman LK, Biller BMK, Findling JW, Newell-Price J, Savage MO, Stewart PM et al. The diagnosis of Cushing's syndrome: an endocrine society clinical practice guideline. J Clin Endocrinol Metab. 2008;93(5):1526-40. DOI: 10.1210/jc.2008-0125.

5. Report CC, Saeian S, Ghayumi SM, Shams M. Hypokalemia associated with a solitary pulmonary nodule. Medicine (Baltimore). 2016;95(50):e5046. DOI: 10.1097/ MD.0000000000005046.

6. Isidori AM, Lenzi A. Ectopic ACTH syndrome. Arq Bras Endocrinol Metab. 2007;51(8):1217-25.

7. Munir A, Newell-Price J. Nelson's syndrome. Arq Bras Endocrinol Metab. 2007;51(8):1392-6.

8. Isidori AM, Kaltsas GA, Mohammed S, Morris DG, Jenkins $P$, Chew SL et al. Discriminatory value of the lowdose dexamethasone suppression test in establishing the diagnosis and differential diagnosis of Cushing's syndrome. J Clin Endocrinol Metab. 2003;88(11):5299-306. DOI: 10.1210/jc.2003-030510.

9. Mahmood MM, John K. Hypokalaemia: common but not always benign. Severe, persistent hypokalaemia secondary to ectopic ACTH from a carcinoid tumour. BMJ Case Reports. 2012;2012. pii: bcr-2012-007193. DOI: 10.1136/bcr-2012007193.

10. Nieman LK, Biller BMK, Findling JW, Newell-Price J, Savage MO, Stewart PM et al. The diagnosis of Cushing's syndrome: an endocrine society clinical practice guideline. J Clin Endocrinol Metab. 2008;93(5):1526-40. DOI: 10.1210/jc.2008-0125.

11. Isidori AM, Kaltsas GA, Pozza C, Frajese V, Newell-Price J, Reznek RH, Besser GM. The ectopic adrenocorticotropin syndrome: clinical features, diagnosis, management, and long-term follow-up. J Clin Endocrinol Metab. 2006;91(2):371-7. DOI: 10.1210/jc.2005-1542.

12. Nieman LK, Biller BMK, Findling JW, Murad MH, Newell-Price J, Savage MO, Tabarin A. Treatment of Cushing's syndrome: an endocrine society clinical practice guideline. J Clin Endocrinol Metab. 2015;100;(8):2807-1831. DOI: 10.1210/jc.20151818.

13. Boddaert G, Grand B, Pimpec-Barthes F, Le Cazes A, Bertagna $X$, Riquet $M$. Bronchial carcinoid tumors causing Cushing's syndrome: more aggressive behavior and the need for early diagnosis. Ann Thorac Surg. 2012;94:1823-9. DOI: 10.1016/j. athoracsur.2012.07.022. 